\title{
Performance of Advanced Telecommand Frame Synchronizer under Pulsed Jamming Conditions
}

\author{
Nele Noels, Marc Moeneclaey \\ TELIN, Ghent University, Sint-Pietersnieuwstraat 41, 9000 Gent, Belgium \\ \{nele.noels, marc.moeneclaey\} @UGent.be
}

\begin{abstract}
This paper studies frame synchronization for use with the advanced communication link transmission unit format that was recently proposed for updating the telecommand synchronization and channel coding standard for space applications. With a view to improving the robustness against jamming, future satellite telecommand systems are planning to adopt directsequence spread spectrum modulation and advanced channel coding. Compared to the frame synchronization algorithm specified in the current Consultative Committee for Space Data Systems (CCSDS) recommendation, we consider a longer start sequence and relax the condition for declaring synchronization. We investigate the performance of this algorithm in the presence of jamming, and show that the frame synchronizer can be designed such that the overall system's robustness against pulsed jamming is limited by the robustness of the code rather than the synchronizer.
\end{abstract}

\section{INTRODUCTION AND STATE-OF-THE-ART}

To increase the resilience of satellite telecommand (TC) links against jamming, next generation TC systems are planning to adopt direct-sequence spread spectrum (DSSS) modulation with a very long pseudo-noise spreading code repetition period and a high spreading factor [1], along with advanced channel coding [2], [3].

The only channel coding scheme currently included in the standards and recommendations for TC applications is a modified $\mathrm{BCH}(63,56)$ code. For the next generation uplink CCSDS standard, more advanced channel codes are being put forward [4]. In [2], [3], the codeword error rate (CER) performances of the current $\mathrm{BCH}(63,56)$ and some of the proposed coding schemes are investigated under DSSS modulation in the presence of jamming, assuming perfect synchronization.

Accurate synchronization is an essential prerequisite for reliable channel decoding. The present study focuses on frame synchronization. Frame synchronization in additive white Gaussian noise (AWGN) channels is a well-researched problem (see, for example, [5], [6]). Only recently, several techniques have been compared specifically with a view to application in deep-space communication uplinks in [7]. In contrast, frame synchronization in the presence of jamming has received only limited attention in the literature. Algorithms, specifically designed to operate under jamming conditions, are described and evaluated in [8]-[10]. The usual space telecommand scenario where a known synchronization word is prefixed to a data frame and is itself preceded by a sequence of alternating $+1 /-1$ symbols is considered only in [9]. Unfortunately, the corresponding analysis does not hold for pulsed jamming. We note that the pulsed jammer situation shows many parallels to a block fading channel. With a view to canceling the effect of a varying or inaccurate estimate of the channel gain, [11] considers frame synchronization algorithms for flat fading channels that do not require channel state information. However, the provided analysis focuses on the sensitivity to a time-invariant channel gain estimation error rather than on the effect of time-varying channel conditions (as for pulsed jamming). Standard-specific vulnerability-tojamming-attacks analyses have been reported, for example, in [12]-[14]. However, to the best of our knowledge, a similar study that is representative for the next generation CCSDS TC standard has not been carried out yet.

The outline of this paper is as follows. Section II describes the system under investigation. Section III provides a theoretical performance analysis of the frame synchronizer in the presence of pulsed jamming. Section IV discusses how the frame synchronization error performance impacts the overal frame error rate (FER) of the system, and establishes a coding scheme dependent design criterion for the frame synchronizer. Section $\mathrm{V}$ presents numerical results for the envisaged future TC system. An appropriate value for the design parameter of the synchronizer is selected, and it is shown that the resulting frame synchronizer complies with the requirement imposed by the codes. Section VI summarizes the main conclusions and indicates a direction for future research.

\section{SySTEM DESCRIPTION}

We consider a satellite TC system using Binary Phase Shift Keying (BPSK) and DSSS modulation. A block diagram showing all relevant parts is depicted in Fig. 1.

The structure of the transmitted signal is illustrated in Fig. 2 [15]-[17]. The physical layer of the CCSDS TC protocol stack processes communication link transmission units (CLTUs). Each CLTU is preceded by a symbol acquisition sequence (AS) consisting of a repetition of the $(1,-1)$ symbol pattern. The CLTUs themselves consist of a known start sequence (SS) of length $S$ for frame synchronization purposes, a data sequence hosting a variable number of fixed-length codewords $(\mathrm{CW})$, and an optional tail sequence (TS) which marks the end of the CLTU. We represent the transmitted symbols as $\left\{s_{k}\right\}$, with $s_{k}$ taken from the symbol alphabet $\{-1,1\}$. Without loss of generality, the SS of the considered CLTU is assumed to be $\mathbf{s}_{0}=\left(s_{0}, s_{1}, \ldots, s_{S-1}\right)$.

Spreading is obtained by applying a very long pseudo-noise (PN) chip sequence of length $L$. The bandwidth of the spread signal $s(t)$ is about $\frac{T_{b}}{T_{c}}$ times larger than that of the original BPSK signal, with $T_{\mathrm{b}}$ and $T_{\mathrm{c}}$ denoting the bit interval and the 


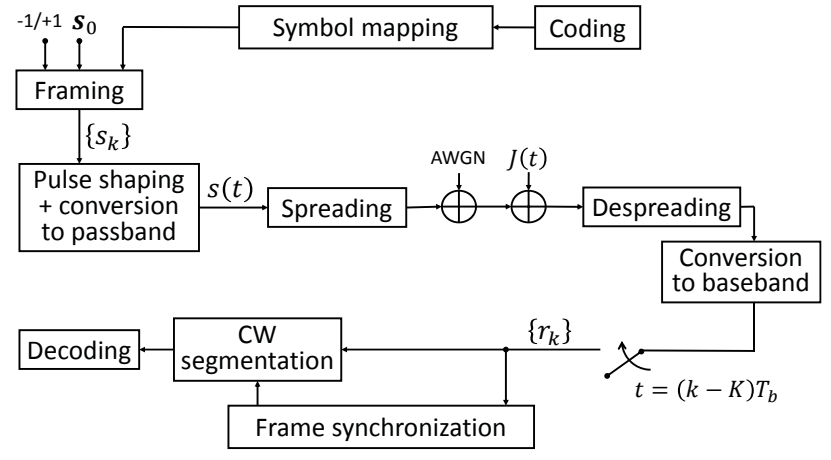

Figure 1. TC system block diagram.

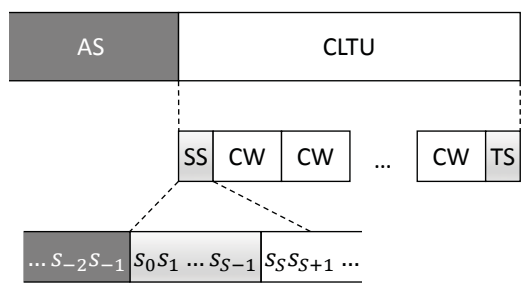

Figure 2. TC physical layer, transmitted signal structure.

chip interval, respectively. In the following we will assume that $T_{b} / T_{c}$ is significantly smaller than $L$ such that the spreading code used for each bit looks as a segment of a long PN sequence (see, e.g., [1]).

The received signal is affected by AWGN with one-sided spectral density $N_{0}$ and by a pulsed jamming signal $J(t)$. The jammer is characterized by a repetition period equal to $Y$ bit intervals, which consists of an active period of $D$ consecutive bit intervals and an inactive period of $Y-D$ bit intervals; the corresponding duty cycle $\rho$ of the jammer is given by $\rho=D / Y$. The boundaries of the repetition periods and the active periods are assumed to coincide with bit boundaries of the useful signal, so that a bit interval from the useful signal is either completely hit or not hit by the jammer. The $Y-D+$ 1 possible starting positions of the active period within the corresponding repetition period are considered equally likely, and independent from one repetition period to the next. During the active period, the jammer power equals $P_{J, p}$, yielding a jammer energy per bit interval equal to $E_{\mathrm{J}, \mathrm{p}}=P_{\mathrm{J}, \mathrm{p}} T_{\mathrm{b}}$ (the subscript 'p' refers to 'peak'); the long-term average jammer power is given by $P_{\mathrm{J}, \text { avg }}=\rho P_{\mathrm{J}, \mathrm{p}}$.

Under the hypothesis of having a large spreading factor $T_{b} / T_{c}$, it can be shown that for a variety of jammer waveforms the jamming contribution to a generic symbol can be modeled by a zero-mean Gaussian random variable [2]. Assuming that perfect synchronization of the PN sequence, the carrier and bit

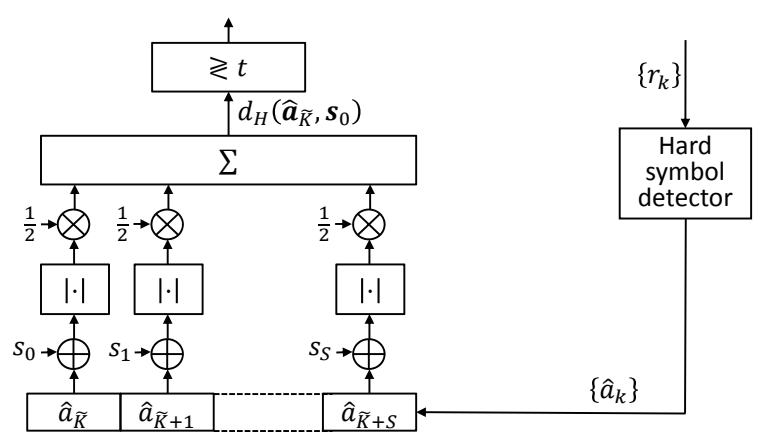

Figure 3. TC frame synchronizer block diagram.

timing has been achieved, the received signal obtained after despreading, conversion to baseband and sampling at the bit rate can be represented as:

$$
r_{k}=\sqrt{E_{s}} s_{k-K}+n_{k}
$$

where the time index $k$ refers to the $k$ th symbol interval observed at the receiver, $E_{s}$ is the received symbol energy, $K$ denotes the unknown delay (in number of bit intervals) of the received signal vis-a-vis the local reference clock, and $\left\{n_{k}\right\}$ are independent real-valued zero-mean Gaussian random variables with time-dependent variance $N_{0 \text {,eq }}(k) / 2$, denoting the combined contribution from the AWGN and the pulsed jammer. When the jammer is active during the $k$ th bit interval we have $N_{0, \text { eq }}(k)=N_{0}+J_{0, \mathrm{p}}$, with $J_{0, \mathrm{p}}=E_{J, \mathrm{p}} T_{\mathrm{c}} / T_{\mathrm{b}}$; when the jammer is inactive during the $k$ th bit interval we have $N_{0, \text { eq }}(k)=N_{0}$. Hence, during its active periods the jammer has the same effect as AWGN with one-sided spectral density $J_{0, p}$.

Frame synchronization is the process of locating the SS of each CLTU, which is required to achieve correct codeword delimitation. It is reasonable to assume that the physical layer operation procedures are properly dimensioned such that the frame synchronizer always starts looking for the SS during the reception of the AS that precedes the CLTU. The frame synchronization procedure described in the recommendations [15]-[17] is illustrated in Fig. 3. It involves performing hard symbol detection on the samples $r_{k}$ from (1), yielding symbol decisions $\hat{a}_{k}=\operatorname{sgn}\left(r_{k}\right)$ of the delayed symbols $a_{k}=s_{k-K}$, with $K$ to be determined by the synchronizer; we define $\hat{\mathbf{a}}_{k}=$ $\left(\hat{a}_{k}, \hat{a}_{k+1}, \ldots, \hat{a}_{k+S-1}\right)$. Next, the synchronizer declares that (the first bit of) the SS occurs at $k=\hat{K}$, when $d_{\mathrm{H}}\left(\hat{\mathbf{a}}_{\hat{K}}, \mathbf{s}_{0}\right) \leq t$, and $d_{\mathrm{H}}\left(\hat{\mathbf{a}}_{\hat{K}-i}, \mathbf{s}_{0}\right)>t$ for $i=1, \ldots, \hat{K} ; d_{\mathrm{H}}(\mathbf{x}, \mathbf{y})$ denotes the Hamming distance between the sequences $\mathbf{x}$ and $\mathbf{y}$. The synchronizer makes no error when the SS is detected at $k=$ $K$.

The current standard recommends a SS of length $S=16$ bit and frame synchronization is declared when at the output of the hard symbol detector a length- $S$ sequence is found which differs from this SS in at most $t$ bits, where either $t=0$ (when 
the $\mathrm{BCH}$ code is used for triple-error detection) or $t=1$ (when the $\mathrm{BCH}$ code is used for single-error correction). Although the methods for detecting the CLTU SS in the case of more advanced coding are yet to be specified, a longer SS ( $S=$ 64 rather than 16) has recently been proposed for the next generation uplink in [18], which improves the resilience of the frame synchronization algorithm against noise, making it also less sensitive to pulsed jamming.

\section{Frame Synchronizer Performance}

In the following, we will determine the performance of the frame synchronizer from Fig. 3 in the presence of pulsed jamming. The parameters $S$ and $t$ will be considered as design parameters.

\section{A. Missed Detection Probability}

For given $t$ and $S$, the missed detection probability $P_{\mathrm{M}}(t, S)$ is the probability that $d_{\mathrm{H}}\left(\hat{\mathbf{a}}_{K}, \mathbf{s}_{0}\right)>t$, where $d_{\mathrm{H}}\left(\hat{\mathbf{a}}_{K}, \mathbf{s}_{0}\right)$ is the number of hard decision errors in the observation of $\mathbf{s}_{0}$. Defining by $\pi_{\mathrm{M}}(l, S)$ the probability that $d_{\mathrm{H}}\left(\hat{\mathbf{a}}_{K}, \mathbf{s}_{0}\right)=l$, $P_{\mathrm{M}}(t, S)$ can be obtained from the recursion $P_{\mathrm{M}}(t, S)=$ $P_{\mathrm{M}}(t-1, S)-\pi_{\mathrm{M}}(t, S)$ for $t=1,2, \ldots, S-1$, with $P_{\mathrm{M}}(0, S)=$ $1-\pi_{\mathrm{M}}(0, S)$. In the following, we point out how to determine $\pi_{\mathrm{M}}(l, S)$ in the presence of jamming.

Defining by $\Pi^{(j)}(l, S)$ the probability that $l$ hard decision errors occur when $j$ out of $S$ observed bit intervals are jammed, and by $P_{j}$ the probability that $j$ out of $S$ observed bit intervals are jammed, we have

$$
\pi_{\mathrm{M}}(l, S)=\sum_{j=0}^{S} \Pi^{(j)}(l, S) P_{j} .
$$

Considering that $l$ errors in the SS correspond to $m$ errors in the $j$ jammed bit intervals and $l-m$ errors in the remaining $S-j$ intervals, with $m \in I(j, l, S)=$ $[\max (0, l+j-S), \min (j, l)]$, we obtain

$$
\Pi^{(j)}(l, S)=\sum_{m \in I(j, l, S)} b\left(m ; p_{1}, j\right) b\left(l-m ; p_{0}, S-j\right),
$$

with $b(i ; p, N)=\left(\begin{array}{c}N \\ i\end{array}\right) p^{i}(1-p)^{N-i}$ denoting the probability mass function of a binomial random variable with mean $N p$ and variance $N p(1-p)$. The probabilities $p_{0}$ and $p_{1}$ in (3) are the hard decision symbol error probabilities $\operatorname{Pr}\left[\hat{a}_{k} \neq a_{k}\right]$ in the absence and presence of jamming, respectively:

$$
\begin{gathered}
p_{0}=Q\left(\sqrt{\frac{2 E_{s}}{N_{0}}}\right), \\
p_{1}=Q\left(\sqrt{\frac{2 E_{s}}{N_{0}+J_{0, p}}}\right),
\end{gathered}
$$

where $Q(x)=\frac{1}{\sqrt{2 \pi}} \int_{x}^{\infty} e^{-t^{2} / 2} d t$ is the complement of the standard Gaussian cumulative distribution function.

Assuming that the position of the first bit interval of the SS is uniformly distributed within the repetition period of the jammer signal, the computation of $P_{j}$ involves determining the number of jammed bit intervals in the SS, for all $Y$ positions of the SS and all positions of the start of the active jammer periods within the jammer repetition periods overlapping with the SS. For conciseness we do not present the equations for $P_{j}$ for arbitrary $(S, D, Y)$ with $D \leq Y$, but rather focus on the following limiting case: when $S \ll \min (D, Y-D)$ and $\rho$ differs from 0 and 1 , the dominant values of $P_{j}$ occur at $j=0$ and $j=S$, with $P_{0} \approx 1-\rho$ and $P_{S} \approx \rho$; in this case we have

$$
\pi_{\mathrm{M}}(l, S) \approx(1-\rho) B\left(l ; p_{0}, S\right)+\rho B\left(l ; p_{1}, S\right) .
$$

\section{B. False Alarm Probability}

We define the false alarm probability $P_{\mathrm{F}, i}(t, S)$, with $i \neq 0$, as the probability that $d_{\mathrm{H}}\left(\hat{\mathbf{a}}_{K-i}, \mathbf{s}_{0}\right) \leq t$. Obviously, $P_{\mathrm{F}, i}(t, S)$ depends on the sequences $\mathbf{s}_{0}$ and $\mathbf{s}_{-i}=$ $\left(s_{-i}, s_{-i+1}, \ldots, s_{K-1-i}\right)$; for $i=1, \ldots, S-1, \mathbf{s}_{-i}$ contains part of the SS and part of the repetition of $(-1,1)$, for $i \geq S$, $\mathbf{S}_{-i}$ is a periodic $(-1,1)$ pattern, alternatingly starting with a '1 ' and a '1' symbol. Defining $\pi_{\mathrm{F}, i}(l, S)$ as the probability that $d_{\mathrm{H}}\left(\hat{\mathbf{a}}_{K-i}, \mathbf{s}_{0}\right)=l$, we can compute $P_{\mathrm{F}, i}(t, S)$ from the recursion $P_{\mathrm{F}, i}(t, S)=P_{\mathrm{F}, i}(t-1, S)+\pi_{\mathrm{F}, i}(t, S)$ for $t=1,2, \ldots$, with $P_{\mathrm{F}, i}(0, S)=\pi_{\mathrm{F}, i}(0, S)$.

Introducing $d_{i}=d_{\mathrm{H}}\left(\mathbf{s}_{-i}, \mathbf{s}_{0}\right)$, the sequences $\mathbf{s}_{0}$ and $\mathbf{s}_{-i}$ agree in $S-d_{i}$ bit intervals and differ in $d_{i}$ bit intervals. Using the law of total probability, we can write

$$
\pi_{\mathrm{F}, i}(l, S)=\sum_{j=0}^{S} \sum_{j^{\prime}=0}^{\min \left(d_{i}, j\right)} \pi_{\mathrm{F}, \mathrm{i}}^{\left(j, j^{\prime}\right)}(l, S) P_{j^{\prime} \mid j ; i} P_{j}
$$

Here, $\pi_{\mathrm{F}}^{\left(j, j^{\prime}\right)}(l, S)$ denotes the probability that $d_{\mathrm{H}}\left(\hat{\mathbf{a}}_{K-i}, \mathbf{s}_{0}\right)=$ $l$ when $j$ out of $S$ bit intervals from the sequence $\mathbf{s}_{-i}$ are jammed and $j^{\prime}$ of these $j$ jammed bit intervals correspond to positions in which $\mathbf{s}_{-i}$ differs from $\mathbf{s}_{0}$. The quantity $P_{j^{\prime} \mid j ; i}$ denotes the probability that $j^{\prime}$ out of the $d_{i}$ bit intervals where $\mathbf{s}_{-i}$ differs from $\mathbf{s}_{0}$ are jammed when in total $j$ out of the $S$ bit intervals of $\mathbf{s}_{-i}$ are jammed. Finally, $P_{j}$ denotes the probability that $j$ out of $S$ bit intervals from the sequence $\mathbf{s}_{-i}$ are jammed.

We have

$$
d_{\mathrm{H}}\left(\hat{\mathbf{a}}_{K-i}, \mathbf{s}_{0}\right)=d_{i}+e_{1}-e_{2},
$$

where $e_{1}$ is the number of hard decision errors in the $S-d_{i}$ bit intervals where $\mathbf{s}_{0}$ and $\mathbf{s}_{-i}$ are the same, and $e_{2}$ is the number of hard decision errors in the $d_{i}$ bit intervals where $\mathbf{s}_{0}$ and $\mathbf{s}_{-i}$ are different. Hence, we obtain

$\pi_{\mathrm{F}, i}^{\left(j, j^{\prime}\right)}(l, S)=\sum_{e_{2}=0}^{d_{i}} \Pi^{\left(j^{\prime}\right)}\left(e_{2}, d_{i}\right) \Pi^{\left(j-j^{\prime}\right)}\left(l-d_{i}+e_{2}, S-d_{i}\right)$,

with $\Pi^{(z)}(x, y)$ the probability that $x$ hard decision errors occur when $z$ out of $y$ observed bit intervals are jammed, defined in (3).

Assuming that the position of the first bit interval of the SS is uniformly distributed within the repetition period of the jammer signal, the computation of $P_{j^{\prime} \mid j ; i}$ involves determining the number of jammed bit intervals in which $\mathbf{s}_{-i}$ differs from $\mathbf{s}_{0}$, for all $Y$ positions of the sequence $\mathbf{s}_{-i}$ and all 
positions of the start of the active jammer periods within the jammer repetition periods overlapping with the sequence $\mathbf{s}_{-i}$. A simplification of (7) results from assuming that if $j$ out of $S$ bit intervals from the sequence $\mathbf{s}_{-i}$ are jammed, then typically about $\frac{j d_{i}}{S}$ of the $d_{i}$ bit intervals in which $\mathbf{s}_{0}$ and $\mathbf{s}_{-i}$ differ are jammed. In that case, $P_{j^{\prime} \mid j ; i}$ approximates the Kronecker delta function $\delta\left(j^{\prime}-\frac{j}{S} d_{i}\right)$ such that (7) reduces to:

$$
\pi_{\mathrm{F}, i}(l, S) \approx \sum_{j=0}^{S} \pi_{\mathrm{F}, \mathrm{i}}^{\left(j,\left\lceil\frac{j d_{i}}{S}\right\rfloor\right)}(l, S) P_{j},
$$

where $\lceil x\rfloor$ denotes the integer closest to $x$. In cases where the $d_{i}$ bit intervals in which $\mathbf{s}_{0}$ and $\mathbf{s}_{-i}$ differ, are more or less evenly spread over the sequence $\mathbf{s}_{-i},(10)$ is a reasonable approximation.

\section{Bound on Synchronization Error Probability}

The synchronization error probability (SEP) is defined as $\operatorname{Pr}[\hat{K} \neq K]$, where $\hat{K}$ and $K$ denote the estimated and the actual position of the SS $\mathbf{s}_{0}$. The frame synchronizer achieves $\hat{K} \neq K$ if and only if $d_{\mathrm{H}}\left(\hat{\mathbf{a}}_{K-i}, \mathbf{s}_{0}\right)>t$ for $i=1, \ldots, K$ and $d_{\mathrm{H}}\left(\hat{\mathbf{a}}_{K}, \mathbf{s}_{0}\right) \leq t$. Hence, the SEP can be bounded as $P_{\mathrm{M}}(t, S) \leq \operatorname{Pr}[\hat{\bar{K}} \neq K] \leq \mathrm{SEP}_{\mathrm{ub}}$, where $\mathrm{SEP}_{\mathrm{ub}}$ denotes the union bound on the SEP:

$$
\mathrm{SEP}_{\mathrm{ub}}=P_{\mathrm{M}}(t, S)+\sum_{i=1}^{K} P_{\mathrm{F}, i}(t, S)
$$

In the numerical results section we will show that in many cases the summation in (11) involving the false alarm probabilities $P_{\mathrm{F}, i}(t, S)$ can be neglected compared to $P_{\mathrm{M}}(t, S)$, in which case we have $\operatorname{Pr}[\hat{\mathrm{K}} \neq \mathrm{K}] \approx P_{\mathrm{M}}(t, S)$.

\section{Frame ERror Rate}

Since erroneously decoded CLTUs are usually discarded at the receiver, the main performance metric for TC applications is the FER. In this section we evaluate the overall FER, which is defined as the ratio of the average number of erroneous CLTUs at the channel decoder output to the number of CLTUs transmitted. A CLTU is considered erroneous when at least one of the codewords contained in the CLTU is affected by decoding errors.

The overall FER can be expressed as

$$
\begin{aligned}
\mathrm{FER} & =\mathrm{FER}_{\hat{K}=K} \operatorname{Pr}[\hat{K}=K]+\mathrm{FER}_{\hat{K} \neq K} \operatorname{Pr}[\hat{K} \neq K] \\
& \leq \mathrm{FER}_{\hat{K}=K}+\operatorname{Pr}[\hat{K} \neq K]
\end{aligned}
$$

where $\mathrm{FER}_{\hat{K}=K}$ and $\mathrm{FER}_{\hat{K} \neq K}$ denote the FER in the absence and presence of frame synchronization errors. The upper bound (12) is tight under normal operating conditions where $\mathrm{FER}_{\hat{K}=K} \ll 1, \mathrm{FER}_{\hat{K} \neq K} \approx 1$ and $\operatorname{Pr}[\hat{K} \neq K] \ll 1$. Denoting by $N_{\mathrm{CW}}$ the number of codewords in a CLTU, we have $\mathrm{FER}_{\hat{K}=K} \leq N_{\mathrm{CW}} \mathrm{CER}$, with CER denoting the codeword error rate in the absence of synchronization errors.

Our aim is to design the frame synchronizer such that the FER is mainly determined by the performance of the code (the term $\mathrm{FER}_{\hat{K}=K}$ in (12)) rather than the synchronizer (the term $\operatorname{Pr}[\hat{K} \neq K]$ in (12)). Hence, reducing $\mathrm{FER}_{\hat{K}=K}$ by using a more powerful code puts a stronger requirement on the frame synchronizer. We consider the operational requirement $\mathrm{FER} \leq \mathrm{FER}_{\max }$ (with $\mathrm{FER}_{\max }=10^{-3}$ as a typical value), and illustrate how this requirement can be met. We denote by $E_{s}\left(N_{\mathrm{CW}} ; D\right)$ the value of the received symbol energy $E_{s}$ which is needed to achieve $\mathrm{FER}_{\hat{\mathrm{K}}=\mathrm{K}}=\frac{1}{2} \mathrm{FER}_{\text {max }}$ under given channel conditions characterized by the parameters $\left(D, \rho, P_{J, p}, N_{0}\right)$; hence, at $E_{s}=E_{s}(1 ; D)$ we have $\mathrm{CER}=\frac{1}{2} \mathrm{FER}_{\text {max }}$. As $\mathrm{FER}_{\hat{\mathrm{K}}=\mathrm{K}}$ increases with $N_{\mathrm{CW}}$ for given $E_{s}, E_{s}\left(N_{\mathrm{CW}} ; D\right)$ must increase with $N_{\mathrm{CW}}$ in order to maintain $\mathrm{FER}_{\hat{\mathrm{K}}=\mathrm{K}}=\frac{1}{2} \mathrm{FER}_{\max }$ for increasing $N_{\mathrm{CW}}$. For every $\left(\rho, P_{J, p}, N_{0}\right)$, we choose one (representative) value of $D$ and select the parameters $t$ and $S$ such that the synchronizer yields $\operatorname{Pr}[\hat{K} \neq K] \leq \frac{1}{2} \mathrm{FER}_{\max }$ for $E_{s}=E_{s}(1 ; D)$; hence, for $N_{C W}=1$ we obtain $\mathrm{FER} \leq \mathrm{FER}_{\max }$ when $E_{s} \geq E_{s}(1 ; D)$. For the same selection of $(t, S)$ and the same $\left(\rho, P_{J, p}, N_{0}\right)$, but a CLTU with $N_{\mathrm{CW}}>1$ and any value of $D$, we obtain $\mathrm{FER} \leq \mathrm{FER}_{\max }$ when $E_{s} \geq \max _{D} E_{s}\left(N_{\mathrm{CW}} ; D\right)$. In this reasoning, we have made use of the fact that $\mathrm{FER}_{\hat{\mathrm{K}}=\mathrm{K}}$ and $\operatorname{Pr}[\hat{K} \neq K]$ are decreasing functions of $E_{s}$.

\section{NUMERICAL RESUlts AND DisCUSSION}

In this section we provide numerical results on the frame synchronization performance in the presence of pulsed jamming. The jamming scenario is characterized by the parameters $\left(D, Y, \frac{E_{s}}{J_{0, p}}\right)$. We focus on next generation TC systems [18] with $S=64$ ( 8 bytes),

$$
\mathbf{s}_{0}=(034776 C 7272895 B 0)_{16},
$$

in hexagonal notation, and the advanced LDPC coding schemes $\mathrm{C} 1$ and $\mathrm{C} 2$ from [4]. The parameter $t$ is to be properly selected. We discuss absolute results as well as the relative contribution of the frame synchronizer to the overall FER. For the CER performance, we rely on results from a previous study [2], with a focus to the case where no interleaving is applied to the coded bits and where the (equivalent) noise variance that is used to calculate the soft decoder input is set equal to the average value $N_{0}+J_{0}$, with $J_{0}=P_{a v g, J} T_{c}=J_{0, p} \rho$. As in [2], a nominal operating SNR of $E_{s} / N_{0}=7 \mathrm{~dB}$ is considered. As a primary region of interest we further select a set of $\left(\rho, \frac{E_{s}}{J_{0, p}}\right)$ values for which at least one of the considered coding schemes can guarantee a minimum level of protection against a dominant pulsed jammer with a period $Y$ equal to 10 times the code word length [2]. From [2], we obtain $\rho \in[0.01,1]$ and $\frac{E_{s}}{J_{0, p}} \in[0,5] \mathrm{dB}$. Taking into account that the ground station typically waits to send the SS until it receives from the on-board receiver an indication that symbol synchronization has been achieved, a rough estimate of the time $K T_{b}$ between the activation of the frame synchronizer and the actual reception of the SS is the round-trip delay of the TC link. Considering a geostationary system with a roundtrip delay of at least $250 \mathrm{~ms}$ and a typical TC communication rate of $4 \mathrm{kbps}$ [19], we obtain $K<K_{\max }$, with $K_{\max }=10^{3}$.

For the SS from (13), it is easily verified that the sequences $\mathbf{s}_{0}$ and $\mathbf{s}_{-i}$, with $i \geq 1$, differ in $d_{i}$ equal to $27,28, \ldots$ or 36 bit intervals that are more or less evenly spread over the 


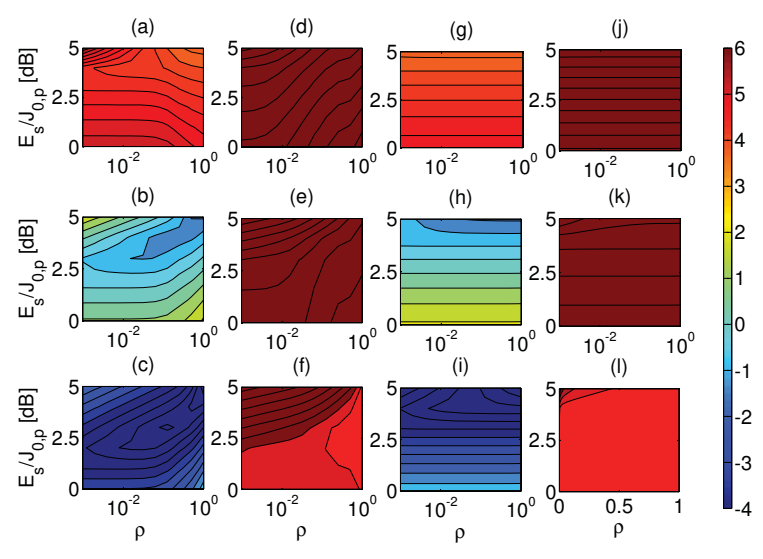

Figure 4. $\log _{10}\left(\frac{P_{M}(t, 64)}{P_{F, i}(t, 64)}\right)$ as a function of $\rho$ and $\frac{E_{s}}{J_{0, p}}$, for $D=40$ ((a)(f)) or $D=640((\mathrm{e})-(\mathrm{l}))$ and for $t=14((\mathrm{a}),(\mathrm{d}),(\mathrm{g}),(\mathrm{j})), t=16((\mathrm{~b}),(\mathrm{e}),(\mathrm{h}),(\mathrm{k}))$ or $t=18$ ((c),(f),(i),(l)).

sequence $\mathbf{s}_{-i}$. The latter justifies the use of (10) to calculate the false alarm probability.

Fig. 4 shows the $\log _{10}$ of the ratio $P_{M}(t, 64) / P_{F, i}(t, 64)$ of the missed detection probability over the false alarm probability over the region of interest for $\left(\rho, \frac{E_{s}}{J_{0, p}}\right)$, and for $d_{i} \in\{27,36\}, D \in\{40,64\}$ and $t \in\{14,16,18\}$. We observe that $P_{M}(t, 64) / P_{F, i}(t, 64)$ does not vary significantly with the value of $D$ and decreases when $d_{i}$ or $t$ increases. For the remainder of this analysis, we select the threshold $t$ equal to 14. It follows from Fig. 4 that this choice of $t$ guarantees that the false alarm probability is at least 3 (= $10 \log _{10} K_{\max }$ ) orders of magnitude smaller than the missed detection probability for all jamming scenarios of practical interest, such that the summation in (11) involving the false alarm probabilities can be safely ingored and the overall SEP is well approximated by the missed detection probability. For larger values of $t$, this approximation no longer holds, which would significantly increase the complexity of the analysis. Choosing a smaller value of $t$ is also not beneficial since it follows directly from the recursive equation in Section III that $P_{M}(t, 64)$, which for $t<14$ approaches the SEP, is a decreasing function of $t$.

Fig. 5 presents the missed detection probability, as a function of the duty cycle $\rho$, for $t=14$ and $E_{s} / \rho J_{0, p}=5$, 7.5, 10 and $15 \mathrm{~dB}$. We observe that:

- For given $\left(E_{s} / N_{0}, E_{s} / J_{0}, D\right)$, there is a value of $\rho$ that maximizes $P_{M}$. This can be explained as follows. From (2)-(5) it is clear that $P_{M}$ increases with increasing $N_{0} / E_{s}$ or $J_{0, p} / E_{s}$. Considering that $\rho$ represents the probability that a symbol is jammed, it further follows that for given $\left(N_{0} / E_{s}, J_{0, p} / E_{s}, D\right) P_{M}$ is an increasing function of $\rho$. The trade-off observed in Fig. 5 is the result of the fact that, for a fixed average value $J_{0}, J_{0, p}$ itself is inversely proportional to $\rho\left(J_{0, p}=J_{0} / \rho\right)$.

- For given $E_{s} / J_{0}$ and given $\rho$, the missed detection probability decreases as $D$ increases. For values of $D$ large as compared to $S, P_{M}$ becomes eventually independent

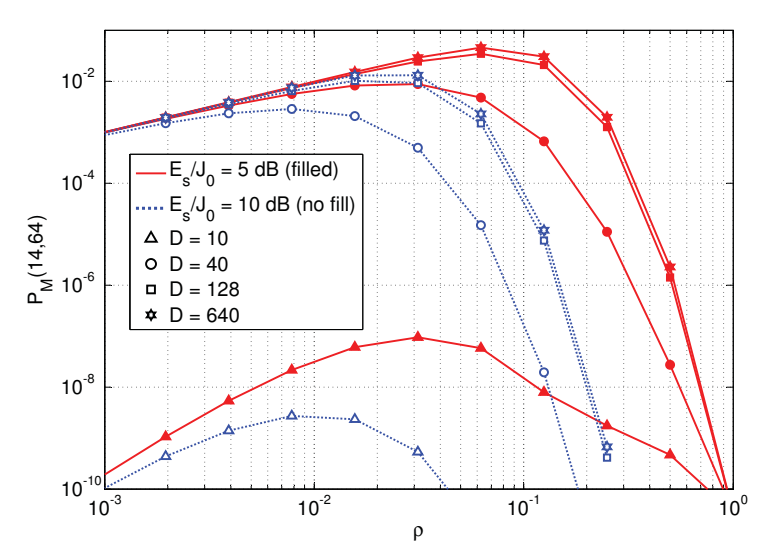

Figure 5. Probability of missed detection as a function of $\rho$ at $E_{S} / N_{0}=7$ $\mathrm{dB}$ for $S=64$ and $t=14$.

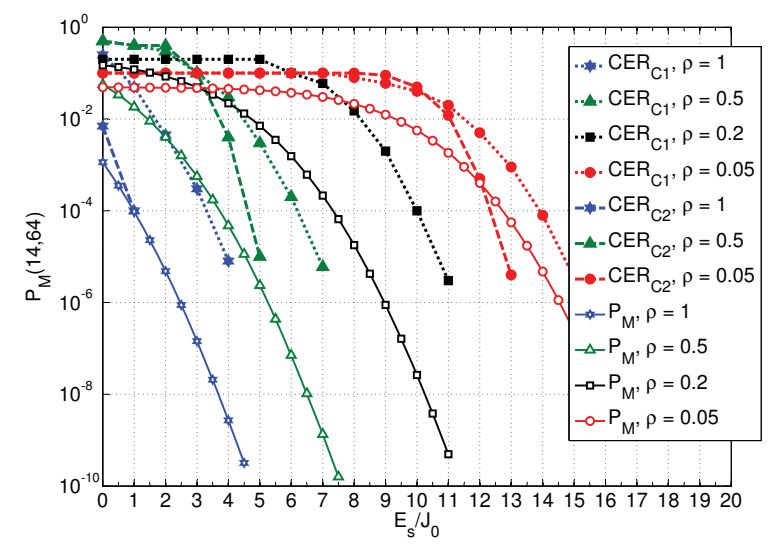

Figure 6. Probability of missed detection for $S=64$ and $t=14$ and codeword error rate (CER) for short LDPC codes, as a function of $E_{s} / J_{0}$ at $E_{s} / N_{0}=7 \mathrm{~dB}$.

of $D$. For these large values of $D$, there is a region of $\rho$ values where $P_{M}$ is approximately equal to $\rho$.

For a given $\left(\rho, \frac{E_{s}}{J_{0, p}}\right)$ in the region of interest and for the selected value of the threshold $t=14$, the frame synchronizer performance is most degraded by pulsed jamming schemes adopting pulses with a very long duration $D$. This justifies that the further investigation of the frame synchronizer performance, namely the comparison with respect to the decoding performance in the absence of frame synchronization errors is narrowed down to the case where, for given $\left(\rho, \frac{E_{s}}{J_{0, p}}\right), D$ goes to infinity. Fig. 6 shows the missed detection probability $P_{M}(14,64)$ (which has been shown to be a close approximation of the frame synchronization error probability for the system under investigation) for $\rho \in\{1,0.5,0.3,0.2,0.1,0.05\}$, $E_{s} / J_{0} \in[0,16] \mathrm{dB}$ and $D$ large as compared to $S$. The figure also displays the numerical CER results (obtained by applying a maximum of 100 sum-product iterations) for two short LDPC codes with rate $1 / 2$ and block lengths of 128 bit (code $\mathrm{C} 1$ ) or 512 bit (code $\mathrm{C} 2$ ), that have been recently 
introduced in the recommendation [4]; the latter results were extracted from [2] and have been obtained for a jammer pulse duration $D$ equal to $\rho Y$ with $Y$ equal to 10 code word lengths. Our results show that, for the considered scenarios, the missed detection probability of the envisaged frame synchronization scheme is

- at least 1 order of magnitude smaller than the CER of $\mathrm{C} 1$, for CER values between $10^{-3}$ and $10^{-6}$.

- smaller (larger) than the CER of C2, for high (low) CER values, with a cross-over point at a CER value smaller than or equal to $5 \cdot 10^{-4}$.

We now verify the design criterion derived in Section IV by posing the following question. Given an operational requirement $\mathrm{FER} \leq \mathrm{FER}_{\max }$, is the frame synchronization error rate smaller than $\frac{1}{2} \mathrm{FER}_{\max }$ for all values of $\frac{E_{s}}{J_{0}}$ larger than or equal to the value of $\frac{E_{s}}{J_{0}}$ which is needed to achieve $C E R=\frac{1}{2} \mathrm{FER}_{\max }$ ? Our results indicate that the answer to this question is

- positive in case of $\mathrm{C} 1$ for a very wide range of operational requirements $\mathrm{FER}_{\max }$.

- positive in case of $\mathrm{C} 2$, provided that $\mathrm{FER}_{\max }$ is larger than or equal to $10^{-3}$, which is the mandatory minimum for TC applications.

- negative in case of $\mathrm{C} 2$, for application in which the maximum allowable total FER, FER max $_{\text {, is significantly }}$ smaller than $10^{-3}$.

Whereas the considered synchronization algorithm is likely to be sufficiently accurate in the presence of jamming as far as the shortest LDPC code $\mathrm{C} 1$ is concerned, for code $\mathrm{C} 2$, the overall FER performance is on the edge of becoming dominated by the performance of the frame synchronizer. The latter may be explained by the difference in slope between the CER of $\mathrm{C} 2$ and the missed detection probability $P_{M}(14,64)$.

\section{CONCLUSIONS AND REMARKS}

We have carried out a theoretical performance analysis of a candidate frame synchronization scheme for future satellite TC systems [18], considering pulsed jamming channels. The considered frame synchronizer was chosen with the objective to minimize the impact of the introduction of the new synchronization scheme to the existing standard.

Our numerical results for the SS from [18] and LDPC codes specified in [4] show that, when the frame synchronizer is properly dimensioned, the minimum signal power that is required to guarantee robustness to a representative set of jamming scenarios is mainly determined by the performance of the code rather than the synchronizer (such that the coding gains over pulsed jamming channels that have been reported in [2] are not jeopardized). It was also shown that the effect of a pulsed jammer, with a given duty cyle and a given power during active periods, on the performance of the considered algorithm is maximum for jammer pulses that are long as compared to the SS.

A possible way to further improve the synchronization error probability under jamming is to adopt more involved
SS detection algorithms. Candidate algorithms are the ones proposed in [7], [11] for AWGN and fading channels. Further research in this direction is left as a topic for future work.

\section{ACKNOWLEDGMENT}

This research has been funded by the Interuniversity Attraction Poles Program initiated by the Belgian Science Policy Office and was carried out under the umbrella of the SatNExIV project. The first author would like to acknowledge the financial support of the Fund for Scientific Research - Flanders.

\section{REFERENCES}

[1] L. Simone, G. Fittipaldi, and I. A. Sanchez, "Fast acquisition techniques for very long PN codes for on-board secure TTC transponders," in Proc. IEEE Military Commun. Conf. (MILCOM ), pp. 1748-1753, 2011.

[2] M. Baldi, F. Chiaraluce, R. Garello, N. Maturo, I. Aguilar Sanchez, and S. Cioni, "Analysis and performance evaluation of new coding options for space telecommand links-part II: jamming channels," Int. J. Satellite Commun. and Networking, vol. 33, no. 6, pp. 527-542, 2015.

[3] P. Martinelli, E. Cianca, and L. Simone, "Comparison of channel codes in presence of pulsed jammers in TT\&C links," in Proc. 7th Advanced Satellite Multimedia Systems Conf. and 13th Signal Processing for Space Commun. Workshop (ASMS/SPSC), pp. 170-173, Sept. 2014.

[4] NASA, CCSDS, Short Block Length LDPC Codes for TC Synchronization and Channel Coding, Oct 2012. Orange Book.

[5] J. Massey, "Optimum frame synchronization," IEEE Trans. Commun., vol. 20, pp. 115-119, Apr. 1972.

[6] M. Chiani and M. G. Martini, "On sequential frame synchronization in AWGN channels," IEEE Trans. Commun., vol. 54, pp. 339-348, Feb. 2006.

[7] S. Pfletschinger, M. Navarro, and P. Closas, "Frame synchronization for next generation uplink coding in deep space communications," in Proc. IEEE Global Commun. Conf. (GLOBECOM), 2015.

[8] R. J. Currie and C. K. Rushforth, "Frame synchronization in the presence of burst jamming," in Proc. IEEE Military Commun. Conf. (MILCOM), vol. 2, pp. 315-319, Oct. 1985.

9] P. F. Driessen, "Performance of frame synchronization in packet transmission using bit erasure information," IEEE Trans. Commun., vol. 39, pp. 567-573, Apr. 1991.

[10] C. K. Rushforth and R. J. Currie, "Frame synchronization in the presence of errors and erasures," IEEE Trans. Aerospace and Electronic Systems, vol. AES-19, pp. 498-505, July 1983.

[11] Y. Liang, D. Rajan, and O. E. Eliezer, "Sequential frame synchronization based on hypothesis testing with unknown channel state information," IEEE Trans. Commun., vol. 63, no. 8, pp. 2972-2984, 2015.

[12] M. Lichtman, J. H. Reed, T. C. Clancy, and M. Norton, "Vulnerability of LTE to hostile interference," in Proc. IEEE Global Conf. Signal and Information Processing (GlobalSIP), pp. 285-288, 2013.

[13] M. Petracca, M. Vari, F. Vatalaro, and G. Lubello, "Performance evaluation of GSM robustness against smart jamming attacks," in Proc. 5th Int. Symp. Commun. Control and Signal Processing (ISCCSP), 2012.

14] M. Barbeau, "Wimax/802.16 Threat analysis," in Proc. 1st ACM Int Workshop on Quality of Service \& Security in Wireless and Mobile Networks, Q2SWinet '05, (New York, NY, USA), pp. 8-15, ACM, 2005.

[15] CCSDS, TC Synchronization and Channel Coding, Sep 2010. 231.0-B-2 Blue Book.

[16] CCSDS, Technical Corrigendum 1 to CCSDS 231.0-B-2, Issued September 2010, Apr 2013. 231.0-B-2 Issue 2 Cor. 1 Blue Book.

[17] CCSDS, TC Synchronization and Channel Coding - Summary of Concept and Rationale, Nov 2012. 230.1-G-2 Green Book.

[18] CCSDS, Next Generation Uplink, Jul 2014. 230.2-G-1 Green Book.

[19] M. Juliato, C. Gebotys, and I. A. Sanchez, "On the specification of symmetric key management parameters for secure space missions," in Proc. IEEE 1st AESS European Conf. on Satellite Telecommun. (ESTEL), 2012. 